\title{
A comprehensive VIKOR method for material selection
}

\begin{abstract}
In engineering design, material alternatives evaluate according to different criteria depending on the objectives of the problem. Performance ratings for different criteria are measured by different units, but in the decision matrix in order to have a valid comparison all the elements must be dimensionless. However, a lot of normalization methods have been developed for cost and benefit criteria, not only there has not been enough attention for engineering design situations in which approaching the target values are desirable but also the available methods have shortcomings. A new version of VIKOR method, which covers all types of criteria with emphasize on compromise solution, is proposed in this paper. The proposed comprehensive version of VIKOR also overcomes the main error of traditional VIKOR by a simpler approach. Suggested method can enhance exactness of material selection results in different applications, especially in biomedical application where the implant materials should possess similar properties to those of human tissues. Five examples are included to illustrate and justify the suggested method.
\end{abstract}

Keyword: Weighting and ranking factors; Performance indices; Selection for material properties 